\title{
Sodium-Benzophenone Ketyl as an Initiator of Anionic Polymerization of Methyl Methacrylate
}

\author{
Masaaki Ogasawara, Hiroshi Yoshida, and Koichiro Hayashi* \\ Faculty of Engineering, Hokkaido University, Kita-ku, Sapporo 060, Japan.
}

(Received July 10, 1974)

\begin{abstract}
The anionic polymerization of methyl methacrylate in tetrahydrofuran initiated by sodium-benzophenone ketyl was studied in rigorously purified systems by means of optical absorption measurements as well as conventional polymerization techniques to elucidate the initiation process involved. It was concluded, on the basis of kinetic behavior of the ketyl anion in reacting with the monomer and the UV absorption of the product polymer, that the addition of the ketyl anion to the monomer forms a propagating carbanion and thus initiates the polymerization. The addition reaction shows an activation energy of $13 \mathrm{kcal} / \mathrm{mol}$ and a rate constant of $1.2 \times 10^{-2}$ $M^{-1} \mathrm{sec}^{-1}$ at $-10^{\circ} \mathrm{C}$. It was also found that no termination reaction occurs, so that the propagating chains are long-lived, though the chain transfer reaction takes place very slowly. The chain transfer reaction is brought about by electron transfer from the propagating carbanion to the benzophenone present in excess in the reaction system to regenerate the initiator.
\end{abstract}

KEY WORDS Anionic Polymerization / Methyl Methacrylate / Benzophenone Ketyl / Radical Anion / Absorption Spectrum / Initiation Mechanism / Chain Transfer / Electron Transfer /

Several studies have been reported on the anionic polymerization of vinyl monomers initiated by a radical anion of aromatic hydrocarbons. $^{1}$ In these, it was well established that the initiation reaction involves the electron transfer from the radical anion to the monomer for the polymerization of styrene and its derivatives. $^{2}$ On the other hand, although the radical anion of aromatic ketones is known to initiate the anionic polymerization of various vinyl monomers, such as methyl methacrylate (MMA), only a few studies have been reported ${ }^{3-7}$ and the initiation mechanisms involved are still ambiguous.

Inoue, et al., ${ }^{3}$ and Smith, et al., ${ }^{4}$ found independently that the benzophenone ketyl anion generated by reducing benzophenone with alkali metals initiated the polymerization of MMA. It was also found that the polymerization showed a typical anionic nature in the copolymerization with acrylonitrile. ${ }^{5}$ However, there have been

* Present address: The Institute of Scientific and Industrial Research, Osaka University, Suita, Osaka 565. two conflicting views on the initiation mechanism of this anionic polymerization. Zilkha, et al. ${ }^{6}$ studied the polymerization of MMA and acrylonitrile with sodium-benzophenone ketyl as initiator in tetrahydrofuran (THF) and concluded from the IR spectra of the obtained polymers that the attachment of the ketyl alkoxide group to the monomer was the initiation step of the polymerization. But, Mengoli and Vidotto $^{7}$ studied the reaction between benzophenone ketyl anion and MMA monomer by polarographic methods and suggested that the initiating entities were formed through electron transfer to the monomer from a dianion which was formed by disproportionation of the ketyl anions.

The primary concern in the present investigation is to uncover the initiation mechanism in the anionic polymerization of MMA with sodiumbenzophenone ketyl as initiator. To this end, the reaction between sodium-benzophenone ketyl and monomer in THF was studied by means of spectroscopic methods in a closed reaction system which was rigorously purified. 
In order to confirm the interpretation of the initiation mechanism, the polymerization was also carried out under the same conditions.

\section{EXPERIMENTAL}

\section{Chemicals}

All the chemicals used were carefully treated to remove all traces of water. Monomeric MMA (Wako Pure Chemical Ind. Co., Ltd.) of special grade was fractionally distilled after washing with sodium hydrosulfate, sodium hydroxide, and distilled water, and rigorously dried on a vacuum line with baked molecular sieves. A measured amount of MMA was transferred into an ampoule having a break seal at the bottom and stored in a Dewar filled with dry ice.

THF (Wako Pure Chemical Ind. Co., Ltd.) of special grade was fractionally distilled over sodium metal, stored over hydrogen calcium, and dried on a vacuum line with sodiumpotassium alloy several times. Benzophenone (Wako Pure Chemical Ind. Co., Ltd.) of special grade was purified by sublimation in vacuo. Sodium metal (Nakarai Chemicals, Ltd.) of guaranteed reagent grade was vacuum-distilled into glass ampoules with a break seal; the insides were thus coated with a fine sodiummirror.

\section{Preparation of Sodium-Benzophenone Ketyl}

The sodium-benzophenone ketyl solution in THF was prepared in the glass apparatus illustrated in Figure 1; attention was paid to its purity and its concentration was carefully controlled. The ampoules were attached to vessel A through break seals, one containing benzophenone and the other a sodium-mirror. Four or six ampoules, each having a break seal $\mathrm{C}$ at the bottom, were connected to vessel B on its periphery through thin tubes. The volumes of both ampoules and tubes were calibrated in advance. The vessels A and B were connected through a glass filter $\mathrm{F}$.

The whole glass apparatus was connected to a vacuum line with the ground joint $G$, evacuated, and flamed thoroughly. A measured amount of THF was transferred into the vessel

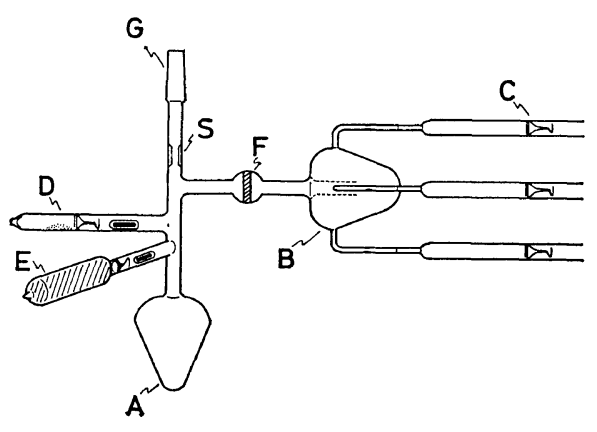

Figure 1. Glass apparatus used for preparation of the solution of sodium-benzophenone ketyl: A and $\mathrm{B}$, vessels; C, break seal; D, benzophenone; $\mathrm{E}$, sodium-mirror; $\mathrm{F}$, glass filter; $\mathrm{S}$, constriction; $G$, ground joint.

A and degassed to $10^{-5}$ torr. The apparatus was then removed from the vacuum line by sealing at the constriction S. Benzophenone was dissolved into THF through the break seal and made contact with the sodium-mirror by introducing a fraction of the solution into the ampoule with sodium in it. This procedure was repeated until a desired amount of benzophenone ketyl $\left(10^{-4} M\right)$ was formed. The sodium-benzophenone ketyl thus prepared was colored deep blue. Although the ketyl anion decayed considerably immediately after the preparation because it reacted with impurities present in the apparatus, it was fairly stable even at room temperature after complete consumption of the impurities. The solution of ketyl anion was transferred from vessel A to vessel B through the glass filter $\mathrm{F}$ and divided into small portions by filling each ampoule to the mark scratched on the calibrated tube. The ampoules were sealed, removed from the apparatus, and stored at liquid-nitrogen temperature. In order to avoid the formation of dianions of benzophenone, an excess amount of benzophenone was used to prepare the ketyl anion. Therefore, some excess benzophenone was necessarily contained in the solution of ketyl anion, though its concentration was very low (lower than the concentration of the feed benzophenone, $1 \times$ $\left.10^{-3} M\right)$. The absence of the dianion was confirmed by measuring the visible absorption spectrum. 
Measurements of the Decay of Sodium-Benzophenone Ketyl

The apparatus for the rapid mixing of initiator with monomer was made of pyrex glass and consisted of three branches in a Y-shape. One of the branches had a quartz cell for optical absorption measurements. The other two branches were used as reservoirs of the ketyl anion solution and the monomer, respectively. First, the cell was kept upright and the ketyl anion solution and MMA were transferred into the reservoirs from ampoules through a break seal. The reactants in the reservoirs were cooled to the desired temperature in a Dry Icemethanol mixture. By turning the apparatus over, the reactants were mixed rapidly and transferred into the cell, which was then set into a precooled Dewar vessel with quartz windows. The decay of the optical absorption of the ketyl anion was monitored at $670 \mathrm{~nm}$; the light from the quartz windows and the cell was analyzed by means of a recording spectrophotometer (Hitachi, EPS-3T). It took only a few seconds to set the optical cell in the spectrophotometer, but a longer time was needed to attain thermal equilibrium in the reacting solution. The time required for the thermal equilibrium was less than $10 \mathrm{sec}$ at a reaction temperature above $0^{\circ} \mathrm{C}$, while it exceeded 20-30 sec at $-20^{\circ}--30^{\circ} \mathrm{C}$. The temperature was measured by a copper-constantan thermocouple positioned close to the optical cell.

\section{Polymerization}

Polymerization was carried out in a reaction apparatus which consisted of an optical cell and a reaction vessel. Before starting the polymerization, the ketyl anion solution was introduced into the reaction apparatus through a break seal and the concentration of the ketyl anion was determined by measuring the intensity of its absorption spectrum $\left(\varepsilon_{\max }=12000 \mathrm{M}^{-1} \mathrm{~cm}^{-1}\right){ }^{8}$ The solution was then transferred into the reaction vessel, cooled to the desired temperature, and mixed with MMA by crushing a break seal. Polymerization was stopped by opening the reaction apparatus and pouring the contents into methanol.

\section{Isolation and Purification of the Polymer}

Polymers precipitated with methanol were filtered, washed with methanol several times, and dried in vacuo. Each polymer was then dissolved into a small amount of THF, filtered to remove tiny pieces of broken glass, reprecipitated with methanol, filtered, and dried to constant weight.

\section{Molecular-Weight Measurements}

The molecular weight and its distribution for each polymer were determined with a gel permeation chromatograph (GPC, Toyo Soda, HLC-301) which was equipped with a column 4 feet long filled with mixed gels. Measurements were made at $20^{\circ} \mathrm{C}$ using THF as the solvent.

\section{RESULTS}

\section{Reaction between Sodium-Benzophenone Ketyl and Monomer}

The ketyl anion formed by reducing benzophenone with sodium in THF is known to have an intense absorption spectrum with a maximum at $670 \mathrm{~nm} .{ }^{9}$ Upon mixing the monomer in the solution of sodium-benzophenone ketyl, the absorption spectrum intensity decreases, as shown in Figure 2. The decay of the spectrum follows first-order kinetics rather than the secondorder one, as indicated in the insert of Figure 2. The decay rate is found to be proportional to the monomer concentration, as illustrated in Figure 3 for the representative reaction temper-

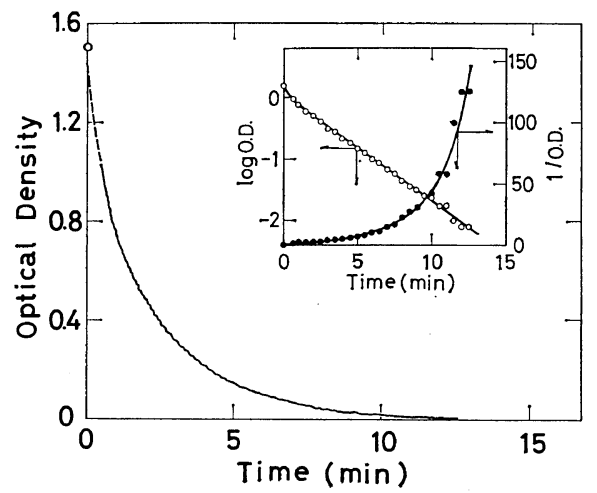

Figure 2. Recorder trace of the decay in optical density of sodium-benzophenone ketyl at $670 \mathrm{~nm}$ after mixing with monomer. Insert represents the first-order and second-order plots of the decay observed. Sodium-benzophenone concentration, $2.5 \times 10^{-4} M$; MMA concentration, $0.43 \mathrm{M}$; temperature, $0^{\circ} \mathrm{C}$. 
ature of $-10^{\circ} \mathrm{C}$.

These results indicate that the ketyl anion disappears in reacting with the monomer, because the monomer concentration is much higher than the concentration of the ketyl anion and is regarded as constant during the reaction. Thus, the second-order rate constant for the reaction between the ketyl anion and the monomer is estimated to be $1.2 \times 10^{-2} M^{-1} \mathrm{sec}^{-1}$ at $-10^{\circ} \mathrm{C}$ from the slope of the linear relationship in Figure 3 and the initial concentration of both the ketyl anion and the monomer. Furthermore, an activation energy of $13 \mathrm{kcal} / \mathrm{mol}$ is found for this reaction from the Arrhenius plot shown in Figure 4.

It may be worthwhile mentioning the effect of benzophenone added in excess into the solu-

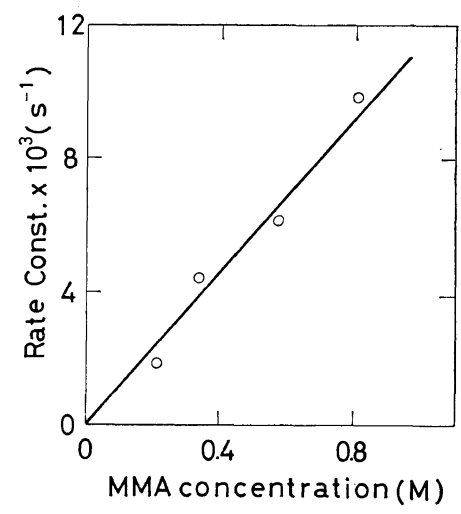

Figure 3. Dependence of the first-order rate constant of the decay reaction of sodium-benzophenone ketyl (corresponding to $k_{i}$ [MMA]) upon the concentration of MMA.

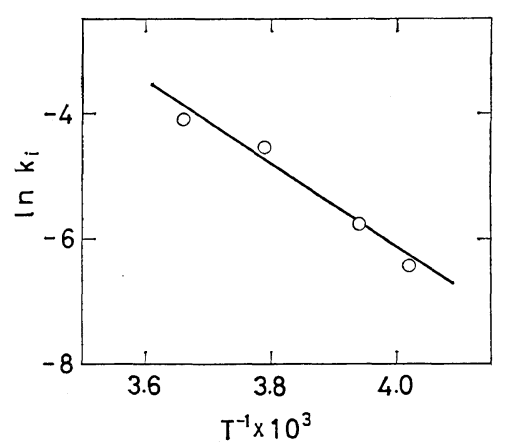

Figure 4. Arrhenius plot of the initiation rate constant, $k_{i}$, for the reaction between sodiumbenzophenone ketyl and MMA.

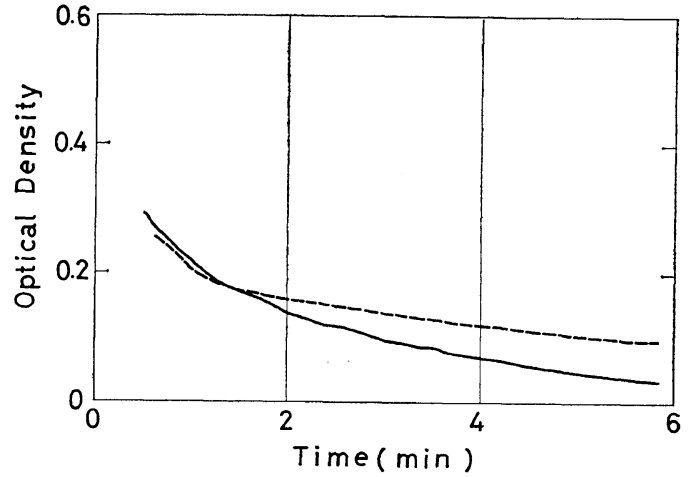

Figure 5. The effect of excess benzophenone on the decay reaction of sodium-benzophenone ketyl with MMA: Solid line, the decay recorded for the "pure" system; dashed line, the decay recorded for the system with $5 \times 10^{-3} M$ of benzophenone intentionally added. Both decays were observed for $1.0 \times 10^{-4} \mathrm{M}$ of sodium-benzophenone ketyl and $0.57 \mathrm{M}$ of MMA at $-10^{\circ} \mathrm{C}$.

tion upon the decay of the ketyl anion. If $5 \times$ $10^{-3} M$ of benzophenone is added intentionally, the decay becomes much slower in the later period of reaction and seems to follow secondorder kinetics, as shown in Figure 5.

The Yield and Analysis of Polymer

The yield of polymer obtained with sodiumbenzophenone ketyl as initiator was found to vary sensitively depending on the purification procedure of the reaction system. To avoid such scattering in the data, a series of results was obtained with the initiator prepared in the same batch. The dependence of the yield upon polymerization time thus obtained is shown in Figure 6, where the decay of the ketyl anion (at the identical conditions of temperature and monomer concentration) is also illustrated for comparison. While the ketyl anion disappears within the first few minutes, the polymerization proceeds linearly with reaction time after an induction period. The dependence of the polymer yield at a fixed polymerization time upon temperature is illustrated in Figure 7 , which has a maximum at about $-10^{\circ} \mathrm{C}$.

No polymer was obtained at temperatures below $-35^{\circ} \mathrm{C}$, where no reaction was actually observed between the ketyl anion and the monomer by spectroscopic measurements. According to detailed studies of the solutions of several 
kinds of alkalimetal-ketyl, paramagnetic dimers and diamagnetic dimers (pinacolate) are also present in the solutions in equilibrium with monomeric ketyl anion. ${ }^{9}$ However, the concentration of these dimeric entities is so low at the present concentration, $10^{-4} \mathrm{M}$, of the monomeric ketyl anion that the contribution of these entities to the polymerization can be ignored. ${ }^{10}$ In addition, the question of whether the poly-

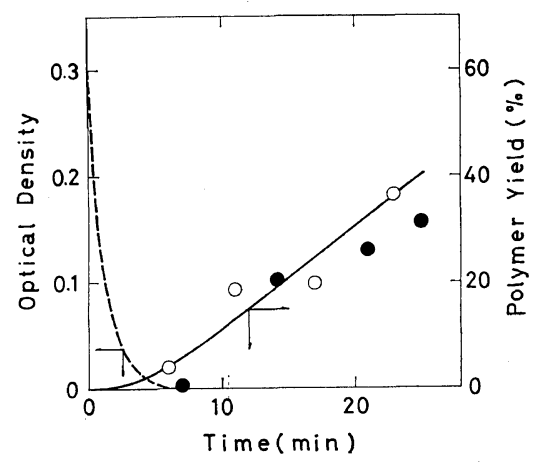

Figure 6. The dependence of polymer yield upon time after mixing MMA with sodium-benzophenone ketyl $(O)$ observed at $-10^{\circ} \mathrm{C}$ for the initiator concentration of $1.11 \times 10^{-4} M$ and the monomer concentration of $0.81 \mathrm{M}$. For comparison, the dashed line shows the decay curve of sodiumbenzophenone ketyl after mixing with MMA under the same conditions as for the polymerization experiment. Polymer yields at $-20^{\circ} \mathrm{C}(\bullet)$ are also plotted to show the linear increase with time for the initiator concentration of $1.41 \times 10^{-4} M$ and the monomer concentration of $0.78 \mathrm{M}$.

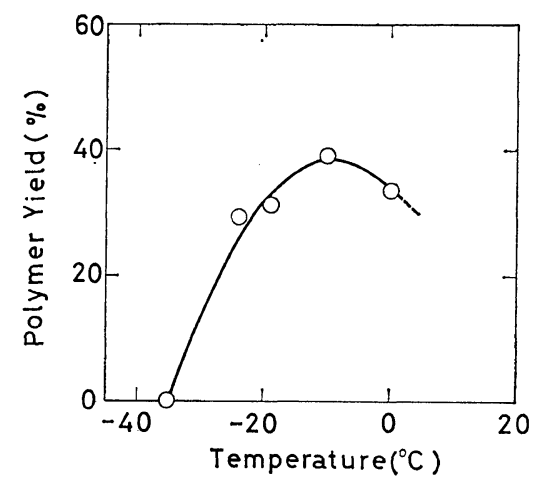

Figure 7. Temperature dependence of the polymer yield obtained at the polymerization time of 30 min after mixing MMA $(0.58 M)$ with sodiumbenzophenone ketyl $\left(0.95 \times 10^{-4} M\right)$. merization could be brought about by any impurities formed during the preparation of the ketyl anion was carefully examined, but no evidence was obtained that the polymerization was due to any entities other than the benzophenone ketyl anion. These observations indicate unambiguously that the polymerization is initiated by the reaction between the sodiumbenzophenone ketyl and the monomer.

The number-average molecular weight, $\bar{M}_{n}$, of each polymer was determined by means of the GPC method. The change in $\bar{M}_{n}$ during polymerization is shown in Figure 8, which indicates that $\bar{M}_{n}$ reaches a constant value in the later period of the polymerization even when the polymer yield is still increasing linearly.

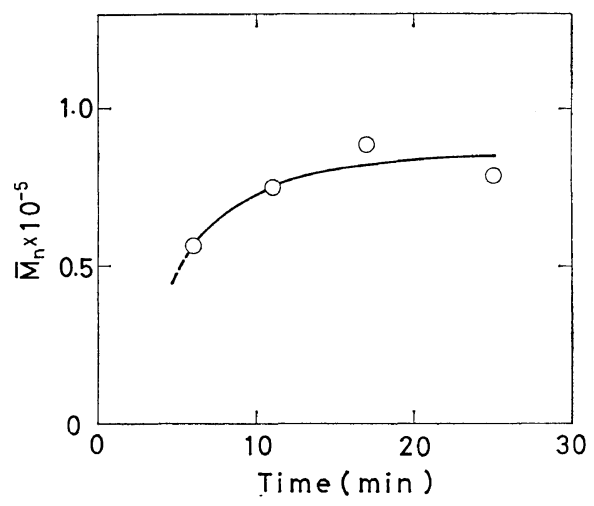

Figure 8. The number-average polymer molecular weight obtained in the polymerization of MMA initiated by sodium-benzophenone ketyl as a function of the polymerization time at $-10^{\circ} \mathrm{C}$ : Initiator concentration, $1.10 \times 10^{-4} \mathrm{M}$; MMA concentration, $0.81 \mathrm{M}$.

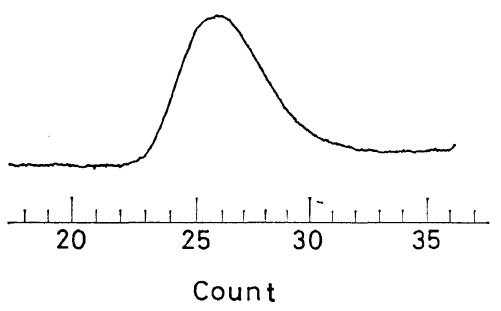

Figure 9. Gel-permeation chromatogram of the polymer obtained in the polymerization of MMA initiated by sodium-benzophenone at $-20^{\circ} \mathrm{C}$ for the initiator concentration of $1.43 \times 10^{-4} M$ and the monomer concentration of $0.83 M$. Measuring temperature, $20^{\circ} \mathrm{C}$; solvent, THF. 
Figure 9 shows a typical result of the GPC for the polymer obtaind at $-20^{\circ} \mathrm{C}$. It indicates a single peak, whose ratio $\bar{M}_{w} / \bar{M}_{n}$ is calculated to be 2 .

The polymer obtained was carefully purified, dissolved in dichloromethane, and subjected to UV absorption measurements. The recorded spectra indicate a weak broad absorption band in the wavelength region of $250-300 \mathrm{~nm}$. On the contrary, poly-MMA obtained from radical polymerization is known to show absorption only at wavelengths shorter than $240 \mathrm{~nm}$, because of the $-\mathrm{COOCH}_{3}$ side group. ${ }^{11}$ This difference is attributed to the diphenyl methoxy group incorporated in the polymer and implies that the attachment of the benzophenone ketyl to the monomer is the initiation step in the present polymerization. The number of diphenyl methoxy groups per polymer chain is roughly estimated to be 1 from the observed optical density and the extinction coefficient (assumed to be $600 \mathrm{M}^{-1} \mathrm{~cm}^{-1}$, half of that of benzpinacol ${ }^{12}$ ) at $260 \mathrm{~nm}$. Although this result does not exclude the possibility that each polymer chain includes two diphenyl methoxy groups because of the uncertainty involved in the estimation procedure, it can show that each polymer chain includes at least one diphenyl methoxy group.

Finally, it should be noted that the polymerization is considerably affected by benzophenone intentionally added into the reaction system. Qualitatively, if one added $5 \times 10^{-3} M$ of benzophenone, only a waxy polymer was obtained with a low yield (about one tenth of the yield in the absence of added benzophenone). However, the data were too scattered to give quantitative results, because of the sensitivity of the polymerization to the purification procedure of the reaction system.

\section{DISCUSSION}

\section{Initiation Mechanism}

It has been shown already that sodiumbenzophenone ketyl acts as an initiator of anionic polymerization of MMA. ${ }^{3-6}$ The present investigation has proved that the reaction between the ketyl anion and the monomer is the initiation reaction of the polymerization. The incorporation of the diphenyl methoxy group into the polymer chain indicates that the most plausible mechanism for the initiation reaction is the addition of the ketyl anion to the monomer,

$$
\begin{aligned}
& \mathrm{Ph}_{2} \mathrm{CO}-, \mathrm{Na}^{+}+\mathrm{CH}_{2}=\mathrm{C}\left(\mathrm{CH}_{3}\right) \mathrm{COOCH}_{3} \\
& \stackrel{k_{i}}{\longrightarrow} \dot{\mathrm{C}}(\mathrm{Ph})_{2} \mathrm{OCH}_{2} \mathrm{C}\left(\mathrm{CH}_{3}\right) \mathrm{COOCH}_{3}, \mathrm{Na}^{+}
\end{aligned}
$$

as suggested by Zilkha, et al. ${ }^{6}$

An alternative interpretation for the reaction between the ketyl anion and the monomer would be the electron transfer from the former to the latter. In the spectroscopic study of the reaction between the radical anion of several aromatic hydrocarbons and MMA by Tobolsky, et $a l,{ }^{13}$ it was found that the electron transfer to MMA occurred from radical anions of biphenyl and naphthalene having a small electron affinity, while that of anthracene, having a large electron affinity, attached to MMA to form a chemical bond. In the present case, benzophenone has a larger electron affinity than MMA and, therefore, the benzophenone ketyl anion is expected to attach to MMA rather than to undergo the electron-transfer reaction. The activation energy, $13 \mathrm{kcal} / \mathrm{mol}$, determined from Figure 4 is a reasonable value for the bond formation and is somewhat too large for the electron-transfer reaction. ${ }^{14}$

The behavior of the ketyl anion observed in the present investigation is different from that reported previously by Mengoli and Vidotto for the reaction between the benzophenone ketyl anion and MMA in dimethylformamide. ${ }^{7}$ They observed that the decay of the ketyl anion followed second-order kinetics, which appears to have been slower than the decay in the present case. Based on these observations and the observed dependence of the decay rate upon the benzophenone present in the reaction system, they suggested that the initiation was

$$
\begin{aligned}
& \mathrm{Ph}_{2} \mathrm{CO}^{2-}+\mathrm{CH}_{2}=\mathrm{C}\left(\mathrm{CH}_{3}\right) \mathrm{COOCH}_{3} \\
& \longrightarrow \mathrm{Ph}_{2} \mathrm{CO}^{-}+\mathrm{CH}_{2}=\mathrm{C}\left(\mathrm{CH}_{3}\right) \mathrm{COOCH}_{3}^{-}
\end{aligned}
$$

which was followed by the reaction between the monomer radical anion thus produced and the monomer to form a propagating carbanion. However, it is very probable that the difference in the decay behavior is caused by the benzo- 
phenone which was present much in excess in the reaction systems in the previous investigation. As a matter of fact, even in the present study, when benzophenone was added in excess to the reaction system, the decay was found to be slowed and to deviate from first-order kinetics. Therefore, it is concluded that the mechanism (2) is excluded for the initiation reaction, at least in THF.

\section{Propagation}

A significant feature in the present polymerization is that the yield of polymer continues to increase linearly with time after the complete disappearance of the initiator, as illustrated in Figure 6. This observation indicates that propagating chain ends have an extremely long lifetime and that actually no termination occurs during the polymerization time studied. The "living" nature of the polymerization gives an interpretation for the induction period observed in the time-polymer yield curves, which is essentially due to the accumulation of propagating chains. The induction period was observed to be longer at lower temperature. This trend is attributed to the slower initiation reaction between the ketyl anion and the monomer, illustrated in Figure 4. The observed single peak in the molecular-weight distribution of the polymer as well as the value of $\bar{M}_{w} / \bar{M}_{n}$ is also consistent with the long lifetime of the propagating chains. The value $\bar{M}_{w} / \bar{M}_{n}$ of 2 is somewhat larger than the value expected for a typical living polymerization, but it is still much smaller than that observed for the anionic polymerization of MMA initiated by organolithium or Grignard reagents. ${ }^{15,16}$

Evidence for the absence of termination and the formation of a living polymer has been obtained by studying the optical absorption spectra of living ends ${ }^{17}$ and the molecular-weight distribution as well as the polymerization kinetics ${ }^{18,19}$ in anionic polymerizations of MMA, mostly at very low temperatures such as $-78^{\circ} \mathrm{C}$. The absorption spectrum of the living chain ends was observed even at temperatures as high as $-30^{\circ} \mathrm{C}$ in toluene. ${ }^{17}$ Recently, Mita, et al., also studied the anionic polymerization of MMA in THF and found that the side reaction to terminate the propagation was absent in the range $-78^{\circ}--65^{\circ} \mathrm{C}^{20}$ These authors claimed that the attack of the propagating carbanion to the carbonyl group of the monomer occurred so efficiently at room temperature as to terminate the polymerization. The present investigation reveals that the propagating carbanion has a long lifetime and the growing polymer appears as "living" in THF even at $-10^{\circ} \mathrm{C}$. However, the decrease in the polymer yield above $-10^{\circ} \mathrm{C}$ shown in Figure 7 suggests the occurrence of a termination reaction at higher temperatures.

We have not yet succeeded in determining directly the concentration of propagating carbanion during the polymerization, mainly because of the overlapping of absorption spectra between the propagating carbanion of MMA and benzophenone. However, the apparent propagation rate constant, $k_{\mathrm{p}}$, can be estimated from the linear slope in Figure 6, if one assumes arbitrarily that the concentration of the propagating chain ends is identical to that of the feed initiator. The $k_{\mathrm{p}}$ value thus estimated is $2 M^{-1} \mathrm{sec}^{-1}$ at $-10^{\circ} \mathrm{C}$, which seems to be unexpectedly small; this will be discussed in a later part of this section.

\section{Chain Transfer}

The data obtained from the analysis of the polymers are summarized in Table I. The ratio of the number of polymer chains to the number of feed initiators reaches as high as 3 for long polymerization times. This observation necessarily implies that the chain-transfer reaction occurs, though slowly, in the present polymerization, even though it has generally not been considered to occur in anionic polymerization of MMA. If one assumes that the free radical ends of polymer formed by the reaction (1) recombine with each other, the value of the ratio is to be multiplied by a factor of 2 . In any case, together with the absence of a termination reaction discussed before, the slow chain-transfer reaction interprets the initial increase and the final plateau in the relationship between the polymer molecular weight and the polymerization time (see Figure 8).

Although the chain transfer in anionic polymerizations is often caused by proton transfer to propagating carbanions, it is not thought to 
Table I. Analysis of polymers obtained by anionic polymerization of methyl methacrylate in THF initiated by sodium-benzophenone ketyl

\begin{tabular}{ccccccc}
\hline $\begin{array}{c}\text { Temperature, } \\
{ }^{\circ} \mathrm{C}\end{array}$ & $\begin{array}{c}\text { Reaction } \\
\text { time, } \\
\text { min }\end{array}$ & $\begin{array}{c}\text { Initiator, } \\
M \times 10^{4}\end{array}$ & $\begin{array}{c}\text { Monomer, } \\
M\end{array}$ & $\begin{array}{c}\text { Polymer } \\
\text { yield, } \\
\%\end{array}$ & $\begin{array}{c}\text { Molecular weight } \\
\bar{M}_{n} \times 10^{-4}\end{array}$ & $\begin{array}{c}\text { Polymer chain } \\
\text { Initiator }\end{array}$ \\
\hline 0 & 20 & 1.61 & 0.81 & 31.5 & 5.32 & 2.98 \\
-20 & 26 & 1.43 & 0.83 & 33.7 & 11.7 & 1.67 \\
-35 & 20 & 1.24 & 0.83 & trace & & 0.33 \\
-10 & 6 & 1.07 & 0.82 & 3.9 & 5.65 & 1.94 \\
-10 & 11 & 1.15 & 0.81 & 19.9 & 7.21 & 1.63 \\
-10 & 17 & 1.10 & 0.81 & 19.7 & 8.86 & 3.43 \\
-10 & 23 & 1.08 & 0.81 & 36.0 & 7.88 & \\
\hline
\end{tabular}

be possible either from monomer or from solvent in the present anionic polymerization of MMA in THF. The most plausible mechanism for the chain-transfer reaction is electron transfer from a propagating chain end to the benzophenone present in excess in the reaction system to regenerate ketyl anion,

$$
\begin{aligned}
& \sim \overline{\mathrm{C}}\left(\mathrm{CH}_{3}\right) \mathrm{COOCH}_{3}, \mathrm{Na}^{+}+\mathrm{Ph}_{2} \mathrm{CO} \\
& \stackrel{k_{\mathrm{tr}}}{\longrightarrow} \sim \dot{\mathrm{C}}\left(\mathrm{CH}_{3}\right) \mathrm{COOCH}_{3}+\mathrm{Ph}_{2} \mathrm{CO}^{-}, \mathrm{Na}^{+}
\end{aligned}
$$

which is able to initiate the polymerization again. All traces of excess benzophenone cannot be removed in the procedure used to prepare the sodium-benzophenone ketyl. The electron transfer from carbanion to benzophenone was previously found to occur for oligo- $\alpha$-methylstyryl anion. ${ }^{21}$

In this respect, it may be informative to study the effect of excess benzophenone on the decay of the ketyl anion upon mixing with the monomer. If one considers the initiation reaction (1) and the chain-transfer reaction (3), the following equation is readily derived under the condition that the termination of the propagating chain ends is ignored:

$$
\begin{aligned}
\frac{\mathrm{d}\left[\mathrm{Ph}_{2} \mathrm{CO}^{-}\right]}{\mathrm{d} t}= & -k_{i}\left[\mathrm{Ph}_{2} \mathrm{CO}^{-}\right][\mathrm{MMA}] \\
& +k_{\mathrm{tr}}\left[\mathrm{P}^{-}\right]\left[\mathrm{Ph}_{2} \mathrm{CO}\right]
\end{aligned}
$$

where $\left[\mathrm{P}^{-}\right]$denotes the propagating chain end. Because of the absence of the termination reaction, $\left[\mathrm{P}^{-}\right]$can be replaced with

$$
\left[\mathrm{P}^{-}\right]=\left[\mathrm{Ph}_{2} \mathrm{CO}^{-}\right]_{0}-\left[\mathrm{Ph}_{2} \mathrm{CO}^{-}\right]
$$

where $\left[\mathrm{Ph}_{2} \mathrm{CO}^{-}\right]_{0}$ represents the initial concen- tration of the ketyl anion. From eq 4 and 5, the decay of the ketyl anion is obtained as

$$
\begin{aligned}
& \frac{\left[\mathrm{Ph}_{2} \mathrm{CO}^{-}\right]}{\left[\mathrm{Ph}_{2} \mathrm{CO}^{-}\right]_{0}}=\left\{1-\frac{k_{\mathrm{tr}}\left[\mathrm{Ph}_{2} \mathrm{CO}\right]}{k_{i}[\mathrm{MMA}]+k_{\mathrm{tr}}\left[\mathrm{Ph}_{2} \mathrm{CO}\right]}\right. \\
& \quad \times \exp \left\{-\left(k_{i}[\mathrm{MMA}]+k_{\mathrm{tr}}\left[\mathrm{Ph}_{2} \mathrm{CO}\right]\right) t\right\} \\
& \quad+\frac{k_{\mathrm{tr}}\left[\mathrm{Ph}_{2} \mathrm{CO}\right]}{k_{i}[\mathrm{MMA}]+k_{\mathrm{tr}}\left[\mathrm{Ph}_{2} \mathrm{CO}\right]}
\end{aligned}
$$

Apparently, the decay follows first-order kinetics with the first-order rate constant of $k_{i}$ [MMA] in the absence of excess benzophenone, as is actually observed in Figures 2 and 3 . In the presence of excess benzophenone, the decay should appear slower because a fraction of the ketyl anion remains for a long time (due to the contribution from the second term in the right-hand side of eq 6). This expectation is consistent with the qualitative observation shown in Figure 5.

From the plateau value of the polymer molecular weight, the chain-transfer rate constant $k_{\text {tr }}$ is determined in principle for the present polymerization by the following relationship:

$$
\overline{\mathrm{DP}}=\frac{k_{\mathrm{p}}[\mathrm{MMA}]}{k_{\mathrm{tr}}\left[\mathrm{Ph}_{2} \mathrm{CO}\right]}
$$

Though the concentration of benzophenone is not very much lower than $10^{-3} M$ in the polymerization system, it cannot be determined exactly. However, the observed molecular weight (see Table I) gives a rough estimate of $k_{\mathrm{tr}}\left[\mathrm{Ph}_{2} \mathrm{CO}\right] /$ $k_{\mathrm{p}}=10^{-3}$ at $-10^{\circ} \mathrm{C}$. The role of benzophenone in the chain-transfer reaction is supported by the fact that the molecular weight of polymer is much lower if a small amount of excess benzophenone is intentionally added. 
Remarks on the Propagation Rate Constant and the Initiation Efficiency

A crucial problem in the present results still remains: the estimated value of $k_{\mathrm{p}}, 2 \mathrm{M}^{-1} \mathrm{sec}^{-1}$, at $-10^{\circ} \mathrm{C}$. Recently, Mita, et al.,${ }^{20}$ and Löhr and Schulz ${ }^{19}$ determined the value of $k_{\mathrm{p}}$ for the propagating carbanion of MMA in THF at very low temperatures. These data imply that the $k_{\mathrm{p}}$ value in the present polymerization might be too small by a factor of $10^{2}$ and suggest that the actual concentration of the propagating chain ends might be very much smaller than that of the feed initiators.

In this connection, the initiation efficiency should be evaluated for sodium-benzophenone ketyl as initiator in the anionic polymerization of MMA. Three distinct results in the present investigation are that (1) the initiator reacts exclusively with the monomer to initiate the polymerization, (2) actually no termination occurs at $-10^{\circ} \mathrm{C}$ or below during the polymerization time studied, and (3) the chain-transfer reaction occurs slowly and regenerates the initiator. If the initiation efficiency is considerably less than unity, the time-polymer yield curve is expected to level off after a few chain transfers, because a considerable fraction of the initiator is deactivated at each subsequent initiation step and the concentration of the propagating chain ends decreases gradually. However, this is not the case, as shown in Figure 6. Although there is no means to evaluate the initiation efficiency directly and exactly in the present polymerization system, it is equal or close to unity. This means that all the reaction between the initiator and the monomer results in the formation of propagating MMA carbanion. The above consideration rules out the possibility that the propagating carbanion thus formed is lost through oligomerization.

It is well known that the common initiators for the anionic polymerization of MMA, such as butyllithium and $\alpha$-methylstyryl carbanion, attack not only the vinyl group but also the carbonyl group of the monomer, so that their initiation efficiency is less than unity. ${ }^{20,22}$ In contrast to them, the benzophenone ketyl anion is thought to attack exclusively the vinyl group of MMA because of its strong basicity. This may be the primary reason why the initiation efficiency is so high in the present polymerization.

Though there was no positive experimental evidence, a fraction of the propagating carbanion may have been destroyed by impurities which could not be eliminated from the polymerization system even though we tried to make the system as pure as possible. Therefore, the propagation rate constant in the present polymerization could be somewhat larger than $2 M^{-1} \mathrm{sec}^{-1}$. Nevertheless, it is still much smaller than the value previously reported for similar reaction systems. ${ }^{19,20}$ Although the presence of benzophenone in the present reaction system may be one of the possible reasons for the difference in the $k_{\mathrm{p}}$ value, a more detailed study is needed for the propagation reaction in the anionic polymerization of MMA initiated by sodium-benzophenone ketyl before we can understand why $k_{\mathrm{p}}$ is so different.

Acknowledgements. The authors are indebted to the Asahi Glass Foundation for the Contribution to Industrial Technology for support of this investigation. They are also grateful to Dr. T. Ichikawa of Hokkaido University for helpful discussions and Mr. Y. Yamamoto of Osaka University for the GPC analysis of the polymers.

\section{REFERENCES}

1. See for example, J. Smid, "Structure and Mechanism in Vinyl Polymerization," T. Tsuruta and K. F. O'Driscoll, Ed., Marcel Dekker Inc., New York, N.Y., 1969, p 374.

2. (a) M. Szwarc, Makromol. Chem., 35, 132 (1960); (b) M. Szwarc, J. Polym. Sci. Part C, 1, 339 (1963).

3. S. Inoue, T. Tsuruta, and J. Furukawa, Makromol. Chem., 36, 778 (1959).

4. S. Smith, J. Polym. Sci., 38, 259 (1959).

5. S. Inoue, T. Tsuruta, and J. Furukawa, Makromol. Chem., 42, 12 (1960).

6. A. Zilkha, P. Neta, and M. Frankel, J. Chem. Soc., 3357 (1960).

7. G. Mengoli and G. Vidotto, Makromol. Chem., 129, 89 (1969).

8. J. F. Garst, D. Walmsley, C. Hewitt, W. R. Richards, and E. R. Zabolotny, J. Amer. Chem. Soc., 86, 412 (1964).

9. N. Hirota, "Radical Ions," E. T. Kaiser and 
L. Kevan, Ed., John Wiley \& Sons, New York, N.Y., 1968, p 35.

10. N. Hirota and S. I. Weissman, J. Amer. Chem. Soc., 86, 2538 (1964).

11. D. L. Glusker, E. Stiles, and B. Yoncoskie, $J$. Polym. Sci., 49, 297 (1961).

12. J. N. Pitts, Jr., B. L. Letsinger, R. T. Taylor, J. M. Patterson, G. Rectenwald, and R. B. Martin, J. Amer. Chem. Soc., 81, 1068 (1959).

13. A. V. Tobolsky and D. B. Hartley, ibid., 84, 1391 (1962).

14. N. Hirota and S. I. Weissman, ibid., 86, 2537 (1964).

15. W. E. Goode, F. H. Owens, and W. L. Myers, J. Polym. Sci., 47, 75 (1960).
16. D. J. Glusker, I. Lysloff, and E. Stiles, ibid., 49, 315 (1961).

17. D. M. Wiles and S. Bywater, ibid., Part B, 2, 1175 (1964).

18. D. Freyss, R. Rempp, and H. Benoît, ibid., Part B, 2, 217 (1964).

19. G. Löhr and G. V. Schulz, Makromol. Chem., 172, 137 (1973).

20. I. Mita, Y. Watanabe, T. Akatsu, and H. Kambe, Polym. J., 4, 271 (1973).

21. Y. Iwakura, F. Toda, H. Katsuki, and $H$. Watanabe, J. Polym. Sci., Part B, 5, 1013 (1967).

22. N. Kawabata and T. Tsuruta, Makromol. Chem., 86, 231 (1965). 